\title{
Richard Poulin
}

Sociologue, professeur titulaire, département de sociologie et d'anthropologie, Université d'Ottawa.

(2008)

\section{"Prostitution \\ et traite des êtres humains: controverses et enjeux."}

Un document produit en version numérique par Jean-Marie Tremblay, bénévole, professeur de sociologie retraité du Cégep de Chicoutimi

Courriel: jean-marie_tremblay@uqac.ca

Site web pédagogique : http://www.uqac.ca/jmt-sociologue/

Dans le cadre de: "Les classiques des sciences sociales"

Une bibliothèque numérique fondée et dirigée par Jean-Marie Tremblay, professeur de sociologie au Cégep de Chicoutimi

Site web: http://classiques.uqac.ca/

Une collection développée en collaboration avec la Bibliothèque

Paul-Émile-Boulet de I'Université du Québec à Chicoutimi Site web: $\underline{h t t p: / / b i b l i o t h e q u e . u q a c . c a / ~}$ 


\section{Politique d'utilisation de la bibliothèque des Classiques}

Toute reproduction et rediffusion de nos fichiers est interdite, même avec la mention de leur provenance, sans l'autorisation formelle, écrite, du fondateur des Classiques des sciences sociales, Jean-Marie Tremblay, sociologue.

Les fichiers des Classiques des sciences sociales ne peuvent sans autorisation formelle:

- être hébergés (en fichier ou page web, en totalité ou en partie) sur un serveur autre que celui des Classiques.

- servir de base de travail à un autre fichier modifié ensuite par tout autre moyen (couleur, police, mise en page, extraits, support, etc...),

Les fichiers (.html, .doc, .pdf, .rtf, .jpg, .gif) disponibles sur le site Les Classiques des sciences sociales sont la propriété des Classiques des sciences sociales, un organisme à but non lucratif composé exclusivement de bénévoles.

Ils sont disponibles pour une utilisation intellectuelle et personnelle et, en aucun cas, commerciale. Toute utilisation à des fins commerciales des fichiers sur ce site est strictement interdite et toute rediffusion est également strictement interdite.

L'accès à notre travail est libre et gratuit à tous les utilisateurs. C'est notre mission.

Jean-Marie Tremblay, sociologue

Fondateur et Président-directeur général, LES CLASSIQUES DES SCIENCES SOCIALES. 
Cette édition électronique a été réalisée par Jean-Marie Tremblay, bénévole, professeur de sociologie au Cégep de Chicoutimi à partir de:

\section{Richard POULIN}

"Prostitution et traite des êtres humains : controverses et enjeux".

Un article publié dans la revue Cahiers de recherche sociologique, no 45, 2008, pp. 135-154. Département de sociologie, UQAM. Montréal : Éditions Liber.

[Autorisation formelle accordée par l'auteur le 13 septembre 2011 de diffuser ce texte dans Les Classiques des sciences sociales.]

Courriel : Richard.Poulin@uottawa.ca

Polices de caractères utilisée: Comic Sans, 10 points.

Édition électronique réalisée avec le traitement de textes Microsoft Word 2008 pour Macintosh.

Mise en page sur papier format : LETTRE US, $8.5^{\prime \prime} \times 11^{\prime \prime}$

Édition numérique réalisée le 11 octobre 2011 à Chicoutimi, Ville de Saguenay, Royaume du Saguenay, Québec. 


\section{Richard Poulin}

Sociologue, professeur titulaire, département de sociologie et d'anthropologie, Université d'Ottawa.

\section{"Prostitution et traite des êtres humains: controverses et enjeux".}

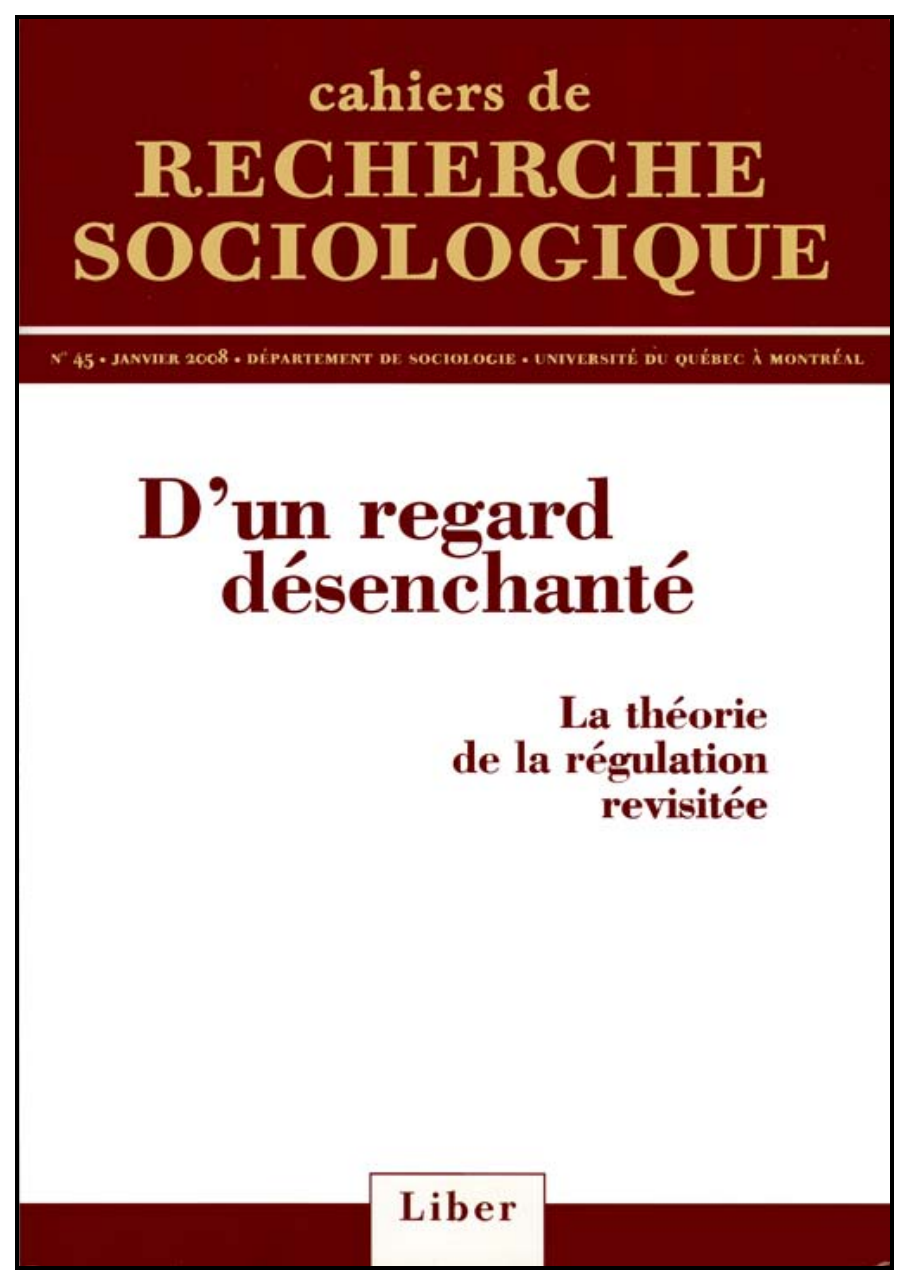

Un article publié dans la revue Cahiers de recherche sociologique, no 45, 2008, pp. 135-154. Département de sociologie, UQAM. Montréal : Éditions Liber. 


\section{Table des matières}

Résumé / Abstract / Resumen

Introduction

L'État des recherches au Canada et au Québec Liberté et droits

Les enjeux autour de la notion de victime

Les débats autour de la convention de Païenne

Autour de la convention abolitionniste

L'offensive réglementariste

En guise de conclusion 
Richard Poulin

Sociologue, professeur titulaire, département de sociologie et d'anthropologie, Université d'Ottawa.

"Prostitution et traite des êtres humains : controverses et enjeux".

Un article publié dans la revue Cahiers de recherche sociologique, no 45, 2008, pp. 135-154. Département de sociologie, UQAM. Montréal : Éditions Liber.

[161]

\section{Résumé}

\section{Retour à la table des matières}

Dans le domaine de la prostitution, de la traite des êtres humains et du trafic des migrants, les perspectives politiques conditionnent en grande partie les recherches, les questions posées, les hypothèses envisagées ainsi que les problématiques. Après avoir examiné la fracture théorique et analytique qui oppose les chercheurs qui définissent la prostitution comme un travail et ceux pour qui elle est une disposition du pouvoir masculin, l'article examine la notion de « victime». Il met en évidence certains de ses enjeux, à travers notamment le processus de négociation menant à l'adoption de la Convention des Nations unies contre la criminalité transnationale organisée. Il fait notamment ressortir que le refus de la notion de victime a pour conséquence la criminalisation des personnes prostituées, qu'elles soient recrutées localement ou à l'étranger. 


\section{Abstract}

\section{Retour à la table des matières}

In the field of prostitution, trafficking in persons and migrants smuggling, political perspectives determine widely researches, asked questions, considered hypotheses as well as problematics. Having examined the theoretical and analytical fracture, which opposes the researchers who define prostitution as a work and those for who it is a disposal of male power, the article examines the "victim's" notion. It puts in light some of the stakes through the process of negotiation leading to the adoption of the United Nations Convention against Transnational Organized Crime. It stands out among others that the refusal of the victim's notion has for consequence the criminalization of the prostituted persons, that they are recruited locally or abroad.

[167]

\section{Resumen}

\section{Retour à la table des matières}

En el campo de la prostitución, la trata de seres humanos y el tráfico de migrantes, las perspectivas sociológicas condicionan en gran parte las investigaciones, las preguntas planteadas, las hipótesis consideradas y las problemáticas. Luego de haber examinado la fractura teórica y analítica que opone a los investigadores que definen la prostitución como un trabajo y a aquellos para los cuales se trata de una disposición del poder masculino, este artículo examina la noción de « víctima». Se pone en evidence lo que está enjuego, particularmente a través del proceso de negociación que lleva a la adopción de la Convención de las Naciones Unidas contra la criminalidad transnacional organizada. Se hace destacar que el rechazo de la noción de víctima tiene por consecuencia la criminalización de las personas prostituidas recrutadas localmente ou en el extranjero. 


\section{Introduction}

\section{Retour à la table des matières}

L'angle sous lequel est analysée la prostitution détermine ce qui est au cour de la problématique : les personnes prostituées et les conditions d'exercice de la «vente» des «faveurs» ou des «services» sexuels - ce qui est la voie traditionnelle des recherches sur la prostitution, mais qui se fait passer pour nouvelle - ou les hommes pour qui et par qui ce système existe. La définition de la prostitution comme un système structuré par les proxénètes qui, contre rémunération, garantissent aux hommes l'accès commercial au corps des femmes, des enfants ${ }^{1}$ et des êtres féminisés déplace l'analyse vers ceux qui organisent le système et profitent de la prostitution d'autrui ainsi que vers la « demande » masculine ${ }^{2}$. Car, qu'elle soit féminine - fillettes, jeunes filles ou femmes de tous âges - ou masculine - garçons, adolescents et jeunes hommes, travestis et transsexuels -, la prostitution est une institution sociale à l'usage quasi exclusif des hommes. C'est une industrie essentiellement vouée au plaisir masculin, ce plaisir étant compris en termes de pouvoir 3 et pas seulement en termes de «satisfaction sexuelle ${ }^{4}$ ».

1 Selon les conventions internationales, un enfant désigne toute personne de moins de dix-huit ans.

2 Les recherches sur les clients prostitueurs, qu'ils soient touristes sexuels ou non, sont relativement rares, comme s'il y avait un pacte du silence autour de cet « acteur » pourtant essentiel. Cela commence à changer.

3 F. Héritier, Masculin/féminin II. Dissoudre la hiérarchie, Paris, Odile Jacob, 2002 ; G. Simmel, Philosophie de Vamour, Paris, Rivages, 1988.

4 C. Legardinier et S. Bouamama, Les clients de la prostitution: l'enquête, Paris, Presses de la Renaissance, 2006 ; S.-A. Mânsson, « Les clients des prostituées et l'image de l'homme et de la masculinité dans la société moderne», Disparition des frontières, trafic des femmes. Actes du 32 e congrès de la Fédération abolitionniste internationale, Copenhague, FAI, 1999. 
[136]

Les chercheurs sont profondément divisés sur la question, tout comme le sont les féministes, les politiciens et la population en général. Une véritable ligne de fracture oppose ceux qui définissent la prostitution comme un « travail » et une vente de "services sexuels», qui veulent la faire reconnaître comme un choix ${ }^{5}$, voire comme une expression de la sexualité féminine ou même comme une subversion du système 6 , et ceux pour qui le système prostitutionnel est une des formes de la violence faite aux femmes, une disposition du pouvoir masculin et une violation des droits humains 7 . Pour l'essentiel, les débats se déploient entre les abolitionnistes, les déréglementaristes et les réglementaristes. Les prohibitionnistes, qui pourtant représentent un courant important dans la société, participent encore peu aux discussions ${ }^{8}$.

5 M. Nengeh Mensah, « Visibilité et droit de parole des travailleuses du sexe. Abolition ou trafic d'un espace citoyen? », Canadian Women Studies/Les cahiers de la femme, vol. 22, nos 3-4, 2003, p. 66-71; voir également K. Kempadoo et J. Doezema (dir.), Global Sex Workers: Rights, Resistance and Redifmition, Londres et New York, Routledge, 1998 ; G. Pheterson, The Prostitution Prism, Amsterdam, Amsterdam University Press, 1996.

6 C. Parent, « Les identités sexuelles et les travailleuses de l'industrie du sexe à l'aube du nouveau millénaire», Sociologie et sociétés, vol. 33, n 1, 1991, p. 5978. Voir aussi F. Delacoste et P. Alexander (dir.), Sex Work: Writings by Women in the Sex Industry, Londres, Virago, 1997.

7 E. Audet, Prostitution. Perspectives féministes, Montréal, Sisyphe, 2005; K. Barry, The Prostitution of Sexuality, New York, New York University Press, 1995 ; S. Jeffreys, The Idea of Prostitution, North Melbourne, Spinifex, 1997 ; Y. Geadah, La prostitution, un métier comme un autre?, Montréal, VLB, 2003.

8 L'abolitionnisme prône la décriminalisation des activités des personnes prostituées, considérées comme des victimes du système de la prostitution et non comme des criminelles et comme les responsables de ce système, et une répression des proxénètes qui exploitent leur prostitution. Il refuse toute réglementation étatique sur les personnes prostituées. Il considère que la traite des êtres humains à des fins de prostitution et l'industrie «nationale» de la prostitution sont étroitement imbriquées. Enfin, il propose une série de mesures de prévention et de sortie de la prostitution ainsi que des services appropriés aux personnes prostituées. Le nouvel abolitionnisme propose également la pénalisation des clients puisqu'ils participent à la prostitution des personnes et en profitent ( $R$. Poulin, Abolir la prostitution, Montréal, Sisyphe, 2006). Le déréglementarisme 


\section{L'ÉTAT DES RECHERCHES AU CANADA ET AU QUÉBEC}

Retour à la table des matières

Selon le point de vue adopté, la prostitution est tour à tour définie comme un «travail du sexe 9 » et un « métier» (le plus vieux, dit-on) ou comme une institution d'oppression des femmes apparue il y a quatre millénaires, en même temps que les marchés, notamment les marchés d'esclaves, et la dégradation du statut des femmes dans les sociétés. Les uns parleront d'inégalité structurelle entre les sexes et de violence sexuée, d'autres d'exploitation sexuelle et même d'esclavage, plusieurs de choix rationnel libre et d'empowerment, quelques-uns de déviance et de criminalité ${ }^{10}$. Certains porteront une attention parti-

préconise la décriminalisation de l'ensemble de l'industrie de la prostitution, en abrogeant du Code criminel tous les articles sur la prostitution. C'est le point de vue de ceux qui considèrent la prostitution comme un travail à déstigmatiser. Les lois du travail leur apparaissent suffisantes pour encadrer le « métier » et protéger les «travailleuses du sexe». Voir, entre autres, $C$. Parent, $C$. Bruckert et $M$. Nengeh Mensah, Pourquoi décriminaliser le travail du sexe, Cybersolidaires, décembre 2004, < http://www.cybersolidaires.org/prostitution/docs/abolos.html >. Les réglementaristes souhaitent la légalisation de certaines formes de prostitution (tout en maintenant une criminalisation d'autres formes). En 2002, le député du Bloc québécois Real Ménard a déposé à la Chambre des communes du Canada le projet de loi C-339 visant à légaliser la sollicitation, les bordels, et proposant la création de zones de tolérance. Le prohibitionnisme interdit la prostitution et criminalise formellement tous ses acteurs, bien que dans la réalité ce soient surtout les personnes prostituées qui subissent la répression. Ce régime juridique est effectif dans une majorité d'États américains et dans les pays musulmans.

9 Voir, entre autres, $C$. Thiboutot, «Lutte des travailleuses du sexe : perspectives féministes », Stella, août 2001, consulté le 15 août 2002 :

http://www.chezstella.org/stella/?q:=node/181.

10 Ce dernier point de vue est désormais largement dépassé au Québec, mais il est encore très fréquent aux États-Unis. Pour en comprendre certains des ressorts, 
culière aux causes de l'entrée dans la prostitution, d'autres limiteront l'analyse aux conditions d'exercice de l'activité des personnes prostituées adultes sans prendre en compte la prostitution des mineurs ${ }^{11}$. Pourtant, les recherches montrent que, au Canada, environ $80 \%$ des adultes en situation de prostitution ont commencé jeunes. L'âge moyen de recrutement tourne autour de quatorze ans ${ }^{12}$. Le même phénomène est noté dans les autres pays capitalistes dominants. Dès lors, l'opinion selon laquelle la grande majorité des personnes en situation de prostitution exercent un libre choix de travail relève de la profession de foi.

La prostitution est perçue par d'aucuns comme une opportunité économique pour les femmes et même comme un travail plus avantageux qu'un autre, non seulement plus rémunérateur, mais également comme l'un des rares lieux où s'exerce un «pouvoir féminin»13. Enfin, des recherches [138] québécoises examinent les liens entre la traite des humains ${ }^{14}$, le tourisme sexuel ${ }^{15}$, le crime organisé ${ }^{16}$, la

voir, entre autres, A. Van Haecht, "Sociologie de la déviance et sociologie de la prostitution », Recherches sociologiques, vol. 8, $n^{\circ} 3,1977$, p. 301-322.

11 Voir respectivement M. Dorais et $P$. Corriveau, Jeunes filles sous influence. Prostitution juvénile et gangs de rue, Montréal, VLB, 2006 ; R. Dufbur, Je vous salue... Le point zéro de la prostitution, Sainte-Foy, Multimondes, 2005 ; R. Poulin, Enfances dévastées. L'enfer de la prostitution, Ottawa, L'Interligne, 2007 ; et F. Shaver, "Sex Work Research. Methodological and Ethical Challenges », Journal of Interpersonal Violence, vol. 20, no 3, 2005, p. 296-319; L. Toupin, « Analyser autrement la prostitution et le trafic des femmes», IVe congrès international des recherches féministes dans la francophonie plurielle: citoyennes sans frontière, université d'Ottawa, 9 juillet 2005.

12 Pour le détail des différentes études et recherches établissant cet âge moyen, voir R. Poulin, Enfances dévastées, op. cit., p. 122-124.

13 D. Brock, Making Work, Making Trouble : Prostitution as a Social Problem, Toronto, University of Toronto Press, 1998 ; L. Ann Jeffrey et G. MacDonald, Sex Workers in the Maritimes Talk Back, Vancouver, UBC Press, 2007 ; L.S. Robinson, "Sex and the City" : la prostitution à l'ère des migrations, mondiales", Recherches féministes, vol. 15, no 2, 2002, p. 41-64.

14 Y. Geadah, op. cit.; R. Poulin, La mondialisation des industries du sexe, Ottawa, L'Interligne, 2004.

15 F. Michel, Voyage au bout du sexe. Trafics et tourismes sexuels en Asie et ailleurs, Québec, Presses de l'université Laval, 2006. 
mondialisation capitaliste néolibérale 17 et la croissance internationale et locale de l'industrie de la prostitution. D'autres atténuent la force de ces liens et circonscrivent la question de la traite des humains à des fins de prostitution à une migration de travailleuses du sexe ${ }^{18}$. Plusieurs essais contradictoires sur le féminisme et la prostitution ont été récemment publiés. Enfin, depuis peu, des recherches s'intéressent aux clients de la prostitution 19.

Les partisans de la déréglementation mènent des recherches sur les conditions d'exercice d'un « travail sexuel marginalisé » et stigmatisé, voire «émotionnel 20 ». Leurs enquêtes, basées sur des échantillons «boule-de-neige » ou " par filière», s'effectuent avec l'aide des associations des «travailleurs du sexe » et sont habituellement limitées à ce réseau particulier 21. Il [139] est donc inapproprié de géné-

16 M. Dorais et P. Corriveau, op. cit.; M. Mourani, La face cachée des gangs de rue, Montréal, éditions de l'Homme, 2006 ; R. Poulin, «Prostitution, crime organisé et marchandisation », Revue Tiers Monde, vol. XLIV, $n^{\circ} 176,2003, \mathrm{p} .735-769$.

17 R. Poulin (dir.), «Prostitution, la mondialisation incarnée», Alternatives Sud, vol. XII, no 3, Louvain-la-Neuve et Paris, Cetri et Syllepse, 2005.

18 J.-M. Chaumont et A.-L. Wibrin, «Traite des Noirs, traite des Blanches : même combat ? », Cahiers de recherche sociologique, no 43, 2007, p. 121-132 ; L. Toupin, «La scission politique du féminisme international sur la question du "trafic des femmes" : vers la "migration" d'un certain féminisme radical ? », Recherches féministes, vol. 15, no 2, 2002, p. 9-40.

19 R. Dufour, op. cit.

20 C. Bruckert et $C$. Parent, « La danse érotique comme métier à l'ère de la vente de soi », Cahiers de recherche sociologique, no 43, 2007, p. 95-107 ; C Bruckert, Taking It Off, Putting It On: Women in the Strip Trade, Toronto, Women's Press, 2002.

21 Voir par exemple C. Bruckert, $C$. Parent et P. Robitaille, Etablissements de services érotiques/danse érotique: deux formes de travail marginalisé, Ottawa, Commission du droit du Canada et ministère de la Condition féminine, 2003. Sous prétexte de répercuter la parole des «travailleuses du sexe», ces recherches se limitent aux personnes liées aux associations de « travailleuses du sexe ». Plusieurs témoignages d'anciennes personnes prostituées, qui refusent d'envisager la prostitution comme un «travail comme un autre», ont été publiés par le site Sisyphe.org. Des « survivantes de la prostitution » ont récemment publié un manifeste contre la reconnaissance de la prostitution en tant que travail \{Survivors of Prostitution and Trafficking Manifesto, CATW, 2005). Les actions, les luttes, les prises de parole de personnes prostituées, ou ayant été prostituées, sont 
raliser les résultats de ces enquêtes à l'ensemble de l'industrie. Toutefois, ces recherches mettent en évidence certaines facettes stigmatisation, criminalisation, problèmes de sécurité et de santé publique, négociations avec les clients, etc. - tout en ne s'intéressant pas à d'autres aspects: conditions et modalités de l'entrée dans la prostitution, agressions sexuelles dans l'enfance, désir de quitter, stress post-traumatique, piètre estime de soi, suicides et tentatives de suicide, etc.

Les recherches à partir d'autres types d'échantillons explorent les liens entre les fugues ou le rejet du foyer familial et les probabilités de l'entrée dans la prostitution: les agressions sexuelles dans l'enfance étant l'une des grandes causes de la fugue des jeunes 22. Aussi, ces recherches montrent un lien étroit entre la probabilité d'intégrer la prostitution et le fait de quitter le milieu familial à un âge précoce, d'avoir été victime de violence sexuelle dans l'enfance 23 et, dans le cas des jeunes hommes, d'avoir subi des discriminations et des violences dues à leur orientation sexuelle ${ }^{24}$. Certaines recherches mettent en évidence le passage de la prostitution occasionnelle de survie à la prostitution régulière ${ }^{25}$. D'autres facteurs sont également examinés

donc diverses et même contradictoires. Aussi, on ne peut que récuser la prétention de tel ou tel courant de pensée de représenter « le point de vue 》 des personnes prostituées/ «travailleuses du sexe» et leur affirmation selon laquelle les autres perspectives et analyses ne tiennent pas compte de leur parole.

22 Voir, entre autres, Régie régionale de la santé et des services sociaux de Montréal-Centre, Le "défi de l'accès » pour les jeunes de la rue, Montréal, La Régie, 1998. Près des deux tiers (63\%) des filles interviewées dans le cadre d'une étude auprès de 479 jeunes sans-abri à Montréal ont été agressées sexuellement.

23 Selon R. Dufour (op. cit.), 85\% des femmes prostituées interviewées ont été agressées sexuellement dans l'enfance. Selon une autre enquête récente menée à Vancouver, $82 \%$ des femmes prostituées ont été victimes d'agressions sexuelles dans l'enfance (M. Farley et J. Lynn, «Prostitution in Vancouver: Pimping Women and the Colonization of First Nations Women», dans $C$. Stark et R. Whisnant (dir.), Not for Sale. Feminists Resisting Prostitution and Pornography, North Melbourne, Spinifex, 2004, p. 106-130).

24 M. Dorais, Les travailleurs du sexe, Montréal, VLB, 2003.

25 R. Poulin, Enfances dévastées, op. cit.; E. Roy, N. Haley, P. Leclerc, N. Lemire, J.P. Boivin, J.Y. Frappier et C. Claessens, «Prevalence of HIV Infection and Risk 
pour comprendre le recrutement: classe sociale, appartenance à une ethnie ou à une minorité nationale, pauvreté, croissance des inégalités sociales et banalisation de la prostitution 26 . Enfin, le rôle des gangs criminalises déjeunes dans la prostitution des filles d'âge mineur a fait l'objet de recherches. Ce facteur est relativement récent: ces gangs ont fait leur apparition au Canada dans les années 1980 et ont investi les industries du sexe dans les années 1990.

[140]

Sont explorées également les violences subies par les personnes prostituées, tant de la part des proxénètes que des prostitueurs. Les taux d'agression physique et sexuelle ainsi que les taux de mortalité sont très élevés. Les femmes prostituées au Canada connaissaient, au début des années 1990, un taux de mortalité quarante fois supérieur à la moyenne nationale et risquaient vingt fois plus l'assassinat 27 . Ces taux sont peut-être encore plus élevés: les femmes prostituées seraient de soixante à cent vingt fois plus souvent agressées physiquement et victimes d'assassinat que tout autre groupe social ${ }^{28}$. Chez les escortes, les tentatives de suicide et les taux de suicide sont les plus élevés au pays, toute catégorie sociale confondue.

D'autres recherches examinent les liens entre les infections transmises sexuellement, la consommation de drogues et d'alcool et la prostitution. La prostitution est généralement associée à la toxicomanie. Un facteur a pourtant été mis en évidence : la prévalence de l'usage de drogues est sensiblement plus élevée chez les personnes prostituées que chez les non-prostituées, mais l'abus de drogues suit géné-

Behaviors among Montreal Street Youth», International Journal Of STD and AIDS, no 11,2000 , p. 241-247.

26 Pour une synthèse des recherches sur ces facteurs, voir R. Poulin, La mondialisation des industries du sexe, op. cit. R. Dufour (op. cit.) met en évidence la normalisation de la prostitution dans l'entourage familial et résidentiel comme facteur propice à l'intégration dans cette industrie. Ce facteur concernerait $15 \%$ des personnes prostituées interviewées.

27 M.A. Baldwin, «Split at the Root: Prostitution and Feminist Discourses of Law Reform », Yale Journal of Law and Feminism, vol. 5, no 1, 1992, p. 47-120.

28 J. Lowman, « Victims and the Outlaw Status of (Street) Prostitution in Canada », Violence Against Women: An International and Interdisciplinary Journal, vol. 6, no 9, 2000, p. 987-1011. 
ralement l'entrée dans la prostitution plutôt qu'elle ne la précède ${ }^{29}$. De toute évidence, les drogues permettent aux personnes prostituées de supporter leur prostitution. Mais la dépendance créée les entraîne à poursuivre sinon accélérer les activités prostitutionnelles dans des conditions de plus en plus risquées et où l'entraide est quasiment inexistante 30 . Enfin, certains liens entre l'injection forcée de drogues dures et le « formatage » à la prostitution ont été mis en évidence par des organisations qui oeuvrent sur le terrain.

Un certain nombre de recherches récentes soulignent la stigmatisation sociale dont sont victimes les personnes prostituées. Le harcèlement policier et judiciaire, la criminalisation et les amendes qui en découlent forcent les personnes prostituées inculpées à multiplier les passes, ce qui fait du système judiciaire à la fois un complice et un responsable de la prostitution. Les préjugés et la méconnaissance entraînent la méfiance envers la parole et le vécu des personnes prostituées. Leurs plaintes pour viol, agressions physiques et sexuelles sont rarement prises en compte par le système (hôpitaux, police, justice, etc.), qui les minimise et les interprète souvent comme faisant partie des « risques du métier». Enfin, on note la rareté et l'inadéquation des services qui leur sont destinés.

[141]

Puisque la majorité des personnes prostituées souffrent d'un état de stress post-traumatique 31 , les tribunaux devraient les considérer comme des victimes et non uniquement comme des témoins, et, à ce titre, elles pourraient bénéficier d'un support et d'un accompagnement. Elles font en général de «mauvais témoins»: elles ont des trous de mémoire, se contredisent, s'effondrent. Pour le juge, elles paraissent de "mauvaise foi 32 ». C'est qu'elles revivent les situations qui ont provoqué chez elles un stress intense. Ne pas tenir compte de

29 S.F. McClanahan, G.M. McClelland, K.M. Abram et L.A. Teplin, « Pathways into Prostitution among Female Jail Detainees and Their Implications for Mental Health Services », Psychiatric Services, vol. 50, no 12, 1999, p. 1606-1613.

30 D'après les témoignages de femmes et de transsexuels prostitués de rue à Ottawa.

31 M. Farley et J. Lynn, art. cité.

32 M. Dorais et P. Corriveau, op. cit. 
cette réalité, c'est favoriser systématiquement le prostitueur et le proxénète.

La normalisation sociale de la prostitution et sa reconnaissance comme travail auraient pour effet de jeter les bases d'une « déstigmatisation » et d'en finir avec les discriminations. Les abolitionnistes considèrent que la réglementation de la prostitution signifie dans les faits un renforcement de l'isolement social et de la stigmatisation. Les gouvernements réglementaristes relèguent les personnes prostituées dans des zones de tolérance, généralement loin des regards (souvent dans des quartiers industriels), et d'autant plus dangereuses, et préconisent l'enfermement dans des bordels sous contrôle des proxénètes qui opèrent en toute légalité, tout en criminalisant les personnes prostituées qui ne sont pas en règle, surtout celles qui sont visibles (sur le trottoir).

\section{LIBERTÉ ET DROITS}

Retour à la table des matières

Les débats sur le «libre choix» de la prostitution ou non sont internationaux et opposent des visions philosophiques et éthiques contradictoires ${ }^{33}$. Ils reprennent le vieux débat entre liberté et déterminisme. Concernant la prostitution, ces débats sont relativement récents. Ce n'est qu'en 1995, lors de la conférence de Pékin, qu'est apparu dans un document international le concept de prostitution «forcée » et « volontaire». Depuis, il structure en grande partie les discours.

L'idée de consentement renvoie, pour les partisans du « travail du sexe», à celle de «libre disposition de son corps» (le «droit» de dire « je charge tant $34 »)$, ce que contestent ceux pour qui il s'agit

33 Voir, entre autres, G. Fraisse, Du consentement, Paris, Seuil, 2007 ; M. Marzano, Je consens, donc je suis..., Paris, PUF, 2006 ; R. Ogien, «L'incohérence des critiques morales du consentement», Cahiers de recherche sociologique, no 43, 2007, p. 133-140.

34 M. Nengeh Mensah, art. cité. 
d'un détournement de la revendication féministe et du délestage de sa visée émancipatrice. Au «droit» à la prostitution des uns est opposé le « droit» de ne pas être prostituée des autres.

Les déréglementaristes partagent avec les réglementaristes l'idée que la prostitution est un «droit» ou une « liberté ». Contrairement à la première [142] vague réglementariste, qui s'appuyait essentiellement sur une vision hygiéniste pour imposer l'enfermement en bordels agréés, c'est désormais au nom du «droit» des personnes de «se» prostituer et du «droit» de «partager » les revenus de leur prostitution avec les proxénètes - qui n'en sont plus, selon les nouvelles lois - que des États européens et du Pacifique Sud ont légalisé (et par le fait même mis hors la loi) certaines formes de prostitution. Jamais ne furent invoqués le « droit» des proxénètes d'exploiter la prostitution d'autrui et celui des hommes à l'accès marchand au sexe des femmes. Le «droit» des unes à la prostitution implique pourtant le «droit» des autres à l'exploiter (proxénète) ou à en user en toute impunité (prostitueur).

Enfin, la réduction de la question de la prostitution à une question de «droit» ou de "liberté » pose d'autres questions: l'égalité en droit dans les sociétés libérales camoufle les inégalités sociales tout en les instituant 35 . Des chercheurs refusent donc d'appréhender la prostitution sous cet angle.

\section{Les enjeux autour de la notion de victime}

\section{Retour à la table des matières}

Les perspectives politiques conditionnent donc en grande partie les recherches, les questions, les hypothèses ainsi que les problématiques sur la prostitution, qui, pour les uns, est un secteur d'activités à normaliser, pour les autres, une perversion immorale inacceptable, du

35 N. Faria, et R. Poulin, Desafíos do livre mercado para o feminismo, Sao Paulo, SOF /Cadernos Sempreviva, 2006. 
moins celle qui est visible 36 , et pour d'autres encore une institution d'oppression des femmes. La personne prostituée sera par conséquent, selon le cas, une travailleuse exerçant un choix rationnel, une criminelle qui détourne les plus vertueux des hommes du droit chemin, ou encore une victime du système proxénète libéral qui ne doit subir ni règlements édictés par l'État, ni criminalisation, ni exploitation par les proxénètes.

La notion de victime prête à toutes les confusions et est souvent détournée de son sens. Pour l'essentiel, c'est un concept juridique, relativement bien défini par les conventions internationales et les lois nationales. Il s'oppose au concept de criminel. Ceux qui l'utilisent sont accusés de « victimiser» les femmes 37 , c'est-à-dire de nier leur autonomie et leur [143] capacité de choix. En conséquence, il leur est reproché d'analyser la prostitution ou la traite des personnes de façon «moralisatrice 38 », ce qui est, bien entendu, condamnable, comme si des concepts comme ceux de la dignité des personnes et de l'inaliénabilité du corps, en partie comme en tout, ne devaient en aucun cas faire partie des analyses. Ce refus de toute considération éthique, notamment celle de l'inaliénabilité du corps, implique une acceptation de

36 Les gouvernements, qu'ils soient réglementaristes, prohibitionnistes ou semi abolitionnistes (notamment la France depuis l'adoption de la loi sur la sécurité intérieure), répriment essentiellement la prostitution de rue, celle qui est visible et donc qui « dérange ». Au Canada, 92\% des affaires juridiques concernant la prostitution sont reliées à l'article 213 du Code criminel, c'est-à-dire l'article sur la communication (le racolage).

37 M. Nengeh Mensah, « Travail du sexe : 14 réponses à vos questions», Stella et le Service aux collectivités de I'UQAM, 2007, http://www.chezstella.org/stella/?q=14reponses. L'argument est rarement utilisé lorsqu'il est question de la prostitution des enfants. Une exception notoire toutefois: J. O'Connell Davidson, Children in the Global Sex Trade, Cambridge, Polity Press, 2007.

38 Pour Stella, «l'amalgame [est] commode entre la prostitution et le trafic des femmes [...]. Lorsqu'il implique des femmes, le trafic des êtres humains n'est considéré que sous l'angle de l'esclavage sexuel, exposant la prostitution en tant qu'épouvantail à mater et favorisant une politique de l'émotion et de l'indignation à courte vue, facilement exploitable» (Stella, «Stella et le débat sur la prostitution », 13 septembre 2002, http://www.cybersolidaires.org/docs/debatTS.html). 
la marchandisation de l'être humain et, en conséquence, son objectivation et son aliénation. Paradoxalement, beaucoup s'opposent à la marchandisation des biens et des services lorsqu'elle concerne le système hospitalier, l'éducation, l'eau, etc., tout en acceptant, sinon en promouvant, la marchandisation des relations humaines, ce qu'est par essence la prostitution des personnes.

Les souteneurs de la prostitution en tant que « travail » nient même l'existence de la «traite des Blanches » ravalée au rang de mythe, tout comme ils minorent systématiquement l'ampleur actuelle de la traite des êtres humains ${ }^{39}$. Cette double négation est étroitement liée à la légitimation de la prostitution. Selon ce point de vue, la « traite des Blanches » montée en épingle aurait permis aux abolitionnistes au tournant du vingtième siècle de marquer des points dans leur campagne contre la prostitution des femmes, c'est-à-dire contre leur autonomie et leur choix. Par conséquent, ils révisent I'histoire et avancent que, depuis plus d'un siècle, les abolitionnistes créent des légendes pour rallier les gouvernements à leur cause. Il n'y aurait pas eu de «traite des Blanches » tout simplement parce que les jeunes femmes prostituées déplacées d'un pays à l'autre et d'un continent à l'autre étaient «consentantes» 40 . Or, étrangement, ce « consentement» était particulièrement obtenu dans les groupes les plus vulnérables, notamment chez les juives d'Europe de l'Est qui ont été les proies les plus nombreuses de cette traite ${ }^{41}$. La traite d'aujourd'hui affecte également de façon disproportionnée les femmes et les enfants de minorités ethniques et nationales.

Actuellement, pour la majorité des victimes des réseaux de la traite, la protection offerte par les gouvernements est de courte durée,

39 C'est le cas de J.-M. Chaumont et A.-L. Wibrin, art. cité.

40 À la veille de la première guerre mondiale, un rapport soumis au Sénat des ÉtatsUnis estimait que la traite internationale des femmes à des fins de prostitution concernait vingt-cinq mille personnes par an (R. Rosen. The Lost Sisterhood. Prostitution in America, 1900-1918, Baltimore, Johns Hopkins University Press, 1983).

41 E. Bristow, Prostitution and Prejudice. The Jewish Fight against White Slaverty, 1870-1939, New York, Schocken Books, 1983. 
quand [144] il y en a ${ }^{42}$. Celles qui portent plainte contre les proxénètes et les trafiquants peuvent bénéficier d'une autorisation provisoire de séjour. Elles sont par la suite généralement rapatriées dans leur pays d'origine. Le plus souvent, elles sont rapatriées d'office et un permis temporaire de séjour leur est accordé ultérieurement au moment du procès 43 . Celles qui refusent de porter plainte, parce que leur vie est en danger ou parce que le réseau qui les a recrutées menace de représailles les proches restés au pays, ne bénéficient souvent d'aucune protection et sont rapatriées manu militari, ce qui, selon l'Organisation internationale pour les migrations, ne fait que nourrir les réseaux de trafiquants, lesquels s'empressent de les remettre sur le marché prostitutionnel mondialisé 44.

Les pays réglementaristes distinguent entre prostitution «forcée » et prostitution « volontaire » et, par conséquent, entre traite à des fins d' "exploitation sexuelle» (synonyme de "prostitution forcée ») et trafic des migrantes «travailleuses du sexe » qui exercent un «travail illicite». Les modalités de la traite leur paraissent une question plus importante que la traite elle-même, ce que mettent en évidence les représentants néerlandais dans les différentes instances internationales et européennes: "S'agissant de la définition de la traite des êtres humains, il importe d'établir une distinction entre "travail illicite" et "prostitution forcée" 45.» Alors, une personne considérée comme « consentante » à sa prostitution n'aura droit à aucune protection particulière et, souvent, sera criminalisée en tant

42 Selon les estimations d'organisations internationales, environ $90 \%$ de la traite internationale implique la prostitution des femmes et des fillettes qui constituent, en 2005, 98\% des victimes de la traite à des fins d' « exploitation sexuelle ». Sur les différentes évaluations de l'ampleur de la traite des personnes, voir R. Poulin, Enfances dévastées, op. cit., p. 25-29.

43 M. Ngalikpima (dir.), L'esclavage sexuel: un défi à l'Europe, Paris, Éditions de Paris et Fondation Scelles, 2005.

44 OIM, Traite des êtres humains, stratégie et activités de VOIM, 86 e session, MC/INF/270, 11 novembre 2003.

45 Mme Zwerver, Pays-Bas, SOC, Migrations liées à la traite des femmes et à la prostitution, rapport de la Commission sur l'égalité des chances pour les femmes et les hommes, Assemblée parlementaire, Conseil de l'Europe, doc. 9795, 23 avril 2003. 
qu'immigrante clandestine, dès lors, elle est une menace à «l'intégrité territoriale» de l'État 46 . Elle n'est donc pas une victime, mais une criminelle - son entrée dans le pays ayant été illégale - dont le « travail » est « illicite».

\section{Les débats autour de la convention de Païenne}

\section{Retour à la table des matières}

Depuis l'adoption de la Convention contre la criminalité transnationale organisée (convention de Palerme) et ses protocoles sur le trafic de migrants et sur la traite des personnes, une terminologie s'est imposée.. Le terme "trafic» (smuggling, en anglais) renvoie au fait de tirer un avantage [145] financier ou matériel de l'« entrée illégale » dans un État « d'une personne qui n'est ni un ressortissant ni un résident permanent de cet État» (article 3a), et celui de «traite» (trafficking) au recrutement, au transport et à l'exploitation. Cette « exploitation » concerne aussi bien la prostitution et d'autres formes non précisées d' « exploitation sexuelle» que la servitude, le travail forcé et le prélèvement d'organes 47 . Le protocole additionnel à la convention de Palerme estime que la traite des personnes peut être condamnable même avec le consentement de la victime, mais impose plusieurs conditions, lesquelles peuvent être interprétées de différentes façons, ce qui permet à des pays qui ont des régimes juridiques très dissemblables d'adhérer au protocole.

Le protocole additionnel à la convention des Nations unies contre la criminalité transnationale organisée visant à prévenir, réprimer et punir la traite des personnes, en particulier des femmes et des enfants, définit la traite dans son article $3:$ «) L'expression "traite des personnes" désigne le recrutement, le transport, le transfert, l'hébergement ou l'accueil de personnes, par la menace de recours ou le recours

46 Ibid.

47 Nations unies, Convention des Nations unies contre la criminalité transnationale organisée, Assemblée générale, A/RES/55/25, 55e session, 8 janvier 2001, http://www.unodc.org/pdf/crime/a_res_55/res5525£pdf. 
à la force ou à d'autres formes de contrainte, par enlèvement, fraude, tromperie, abus d'autorité ou d'une situation de vulnérabilité, ou par l'offre ou l'acceptation de paiements ou d'avantages pour obtenir le consentement d'une personne ayant autorité sur une autre aux fins d'exploitation. L'exploitation comprend, au minimum, l'exploitation de la prostitution d'autrui ou d'autres formes d'exploitation sexuelle, le travail ou les services forcés, l'esclavage ou les pratiques analogues à l'esclavage, la servitude ou le prélèvement d'organes; b) Le consentement d'une victime de la traite des personnes à l'exploitation envisagée, telle qu'énoncée à l'alinéa a du présent article, est indifférent lorsque l'un quelconque des moyens énoncés à l'alinéa $a$ a été utilisé : c) Le recrutement, le transport, le transfert, l'hébergement ou l'accueil d'un enfant aux fins d'exploitation sont considérés comme une "traite des personnes" même s'ils ne font appel à aucun des moyens énoncés à l'alinéa a du présent article; d) Le terme "enfant" désigne toute personne âgée de moins de 18 ans 48 . $\gg$

Il est important de noter que lorsque les réglementaristes citent cet article du protocole additionnel, ils omettent généralement l'alinéa $b$ sur le consentement lequel est « indifférent », c'est-à-dire n'entre pas en ligne de compte, si l'un des moyens énoncés «à l'alinéa $a$ » a été utilisé. Les aliénas $a$ et $b$ ont été l'objet d'une âpre lutte lors des négociations de la convention entre non seulement des représentants de gouvernements, mais également des organisations non gouvernementales parapluies. Le Réseau international des droits humains, animé par la Coalition Against Trafficking in Women (CATW), regroupant cent quarante ONG, y défendait une définition [146] de la traite qui «protège toutes les victimes et non pas seulement celles qui auraient été en mesure de prouver la contrainte 49 ». Dès lors, les personnes victimes de la traite ne devraient plus être perçues comme des criminelles, mais comme des victimes d'un crime. Le réseau a également oeuvré pour que soient assurés des mécanismes de protection pour les

48 Ibid.

$49 \mathrm{~J}$. Raymond, Guide du nouveau protocole sur la traite des Nations unies. Protocole additionnel à la convention contre la criminalité transnationale organisée, visant a prévenir, réprimer et punir la traite des personnes, en particulier des femmes et des enfants, < http://www.ldh-france.org/s, consulté le 20 avril 2004. 
femmes et les enfants victimes de la traite, mais aussi pour que des mesures cohérentes soient intégrées dans la poursuite des trafiquants.

De son côté, le Caucus des droits humains, animé par la Global Alliance Against Trafficking in Women (GAATW), faisait la promotion du « droit des femmes à émigrer pour le travail sexuel » et souhaitait une définition de la traite qui ne mentionne pas la «prostitution». De son point de vue, puisque la prostitution est un travail, il n'y a traite des personnes que lorsqu'il y a usage de la contrainte, autrement dit lorsqu'il y a «travail forcé ». De concert avec les gouvernements ayant légalisé le proxénétisme et réglementé la prostitution, le caucus militait pour restreindre la protection des victimes en limitant le champ de la définition aux seules personnes aptes à prouver l'usage de la force ou de la contrainte dans leur traite. Il refusait notamment les termes d' « incitation » ou d' " abus d'une situation de vulnérabilité », arguant que les « personnes trafiquées » ne pouvaient consentir à leur propre exploitation 50 , et que l'alinéa $b$ sur l'indifférence du consentement dans la traite ne devait pas être introduit dans la définition. De plus, il proposait de remplacer les termes « victimes de la traite » par ceux de «personnes trafiquées 》. Les expressions « victimes» et « exploitation » le heurtaient dans la mesure où il prétend que les activités de l'industrie du sexe ne relèvent pas de l'« exploitation sexuelle». De son point de vue, il y a « exploitation sexuelle» uniquement lorsque les conditions du «travail du sexe» des «personnes trafiquées » sont assimilables à l'esclavage ${ }^{51}$. Sous le prétexte que d'autres formes de traite existent, les réglementaristes refusent que la traite et la prostitution soient intrinsèquement fiées, ce qui pourtant concerne environ $90 \%$ des cas.

50 Au moment des négociations de la convention de Palerme, la Thaillande a proposé que l'on intègre les termes «servitude involontaire » à l'article 3 , ce qui sousentendait qu'il y existait une servitude volontaire et par conséquent non condamnable (M. Marcovich, "La traite des femmes dans le monde», dans $C$. Ockrent (dir.), Le livre noir de la condition des femmes, Paris, XO, 2006, p. 473).

51 Pour un exemple parmi d'autres de ce discours, voir G. Vaz Cabrai, La traite des êtres humains. Réalités de l'esclavage contemporain, Paris, La Découverte, 2006. Vaz Cabrai réduit la portée du protocole contre la traite des personnes à l'exploitation d'une « personne dans des conditions esclavagistes». 
Ce sont avant tout les représentants des gouvernements des États les plus pauvres, les pays d'origine de la traite, qui ont soutenu une définition protégeant les victimes, non limitée à la preuve de l'usage de la force ou de la [147] contrainte, tandis qu'une minorité de pays, principalement occidentaux, les pays de destination, ont défendu une perspective réductrice de la traite 52 .

Néanmoins, une étude attentive de l'article 3 montre les limites d'une interprétation abolitionniste telle que proposée par la CATW et ses alliés. Il est écrit à l'alinéa $c$, qui porte sur la traite des enfants, qu'il y a « traite des personnes » même si « aucun des moyens énoncés à l'alinéa $a$ du présent article » n'a été utilisé. Autrement dit, pour les enfants, en dépit des modalités de leur traite, puisque la notion de consentement est inopérante dans leur cas, la définition de la traite à l'alinéa a n'est pas utile. Cela implique a contrario qu'il n'y a pas de traite des femmes s'il n'y a pas une forme ou une autre de menace ou de recours à la force ou d'autres formes de contrainte, notamment abus d'autorité ou d'une situation de vulnérabilité, ou obtention d'un consentement par une personne exerçant une autorité sur une autre. C'est une réintroduction par la porte de derrière du concept de «prostitution forcée ». Cela explique, à mon avis, pourquoi les gouvernements réglementaristes ont pu ratifier le protocole, malgré leur insatisfaction à l'égard des alinéas a et $b$ de l'article 3.

Selon les règles établies, le protocole ne pouvait être accepté que par consensus. Il était indispensable que tous les pays en approuvent les articles, y compris l'article 3. Le résultat est donc un compromis. Ce qu'atteste l'article 4, «Assistance et protection accordées aux victimes de la traite des personnes », qui laisse la porte ouverte à la non-protection des victimes. Les États peuvent adopter des mesures particulières de protection pour les victimes « lorsqu'il y a lieu et dans la mesure où le droit interne le permet». Même si l'assistance et la protection des victimes ont fait l'objet d'un article, en réalité, rien n'oblige les pays à les mettre en oeuvre. Comme les réglementaristes considèrent que la majorité des «personnes trafiquées» ne sont pas

52 Il s'agit de l'Allemagne, de l'Australie, du Canada, du Danemark, de l'Espagne, de l'Irlande, du Japon, de la Nouvelle-Zélande, des Pays-Bas, du Royaume-Uni, de la Suisse et de la Thaïlande. 
des victimes, rien ne les incite à assurer une assistance ou une protection. En fait, dans les pays qui ont réglementé la prostitution, aucun service particulier n'est offert aux personnes prostituées, qu'elles soient originaires du pays ou étrangères, puisque c'est un travail comme un autre.

Il existe également un lien étroit entre les protocoles sur la traite des personnes et le trafic des migrants. Les victimes de la traite sont souvent confondues avec les migrantes clandestines. Le réseau avait demandé qu'une clause de non-refoulement soit incluse dans le protocole sur le trafic des migrants, afin que ces derniers puissent trouver un refuge et une protection et ne soient pas systématiquement expulsés vers le pays d'origine ou de transit 53 . Alors même que l'immigration devient plus restrictive et [148] discriminatoire, qu'elle est de plus en plus criminalisée dans un contexte où les voies légales sont rendues difficiles et complexes, si ce n'est très onéreuses, les trafiquants deviennent les principaux bénéficiaires de la migration internationale. Les migrants se tournent vers eux. Dans les pays de destination, les trafiquants orientent les femmes et les enfants vers les réseaux qui alimentent les industries du sexe ou le marché du travail clandestin. Le caucus, qui assimile la prostitution à un travail et la traite des personnes à un trafic de migrants, qui plus est à une « migration des travailleuses du sexe », est resté indifférent à cet aspect, ne proposant aucune mesure particulière de protection, acceptant d'emblée leur criminalisation.

La légalisation du proxénétisme et la réglementation de la prostitution entraînent fatalement une légitimation de certaines formes de la traite. En même temps, elles criminalisent: les personnes prostituées, qui ne sont pas en règle, sont victimes de répression tandis que les victimes de la traite à des fins de prostitution doivent prouver l'usage de la force ou de la contrainte pour espérer obtenir une protection de l'État réglementariste. L'opposition entre prostitution «forcée » et prostitution « volontaire » sert d'alibi pour pénaliser les personnes en

53 Deux pays d'Amérique latine ont signé le protocole contre la traite des personnes tout en refusant de signer le protocole contre le trafic de migrants, ce dernier ne disposant pas des mêmes mécanismes de protection des victimes que ceux contre la traite. 
situation d'illégalité qui, contrairement à celles qui se trouvent en situation légale, sont victimes d'une prostitution dite " contrainte » car criminelle selon les règles édictées par ces régimes.

Sans l'intervention du Réseau international des droits humains dans les négociations de la convention de Palerme, les réglementaristes auraient probablement imposé leurs thèses et mis de côté la notion de victime.

\section{Autour de la convention abolitionniste}

\section{Retour à la table des matières}

D'un coté, la CATW assure que la Convention contre la criminalité transnationale organisée s'insère dans le corpus des instruments internationaux des droits humains, en filiation avec la Convention pour la répression de la traite des êtres humains et de l'exploitation de la prostitution d'autrui (ou convention aboHtionniste de 1949) 54 , la Convention relative aux droits des enfants et la Convention sur l'élimination de toutes les formes de discrimination à l'égard des femmes (CEDEF) 55 . L'article 6 de la CEDEF demande aux États de prendre «toutes les mesures appropriées [...] pour supprimer, sous toutes leurs formes, le trafic des femmes et l'exploitation de la prostitution des femmes 56 ».

De l'autre côté, les réglementaristes soutiennent que la convention abolitionniste de 1949 est obsolète. Par exemple, le Parlement européen a [149] adopté une résolution le 9 mai 2000 qui, dans ses considérants, assure que «les instruments juridiques internationaux existants, notamment la Convention des Nations unies relatives à l'abolition de la prostitution, l'exploitation de la prostitution des êtres hu-

54 Disponible en ligne, http://www.urihclir.ch/french/html/menu3/b/-33_fr.htm.

55 CATW, «Victoire à Vienne», The New West Indian, 5 mars 2001, http://www.awigp.com/defeult.asp?numcat=sextour6, consulté le 22 avril 2004.

56 Nations unies, Convention sur l'élimination de toutes les formes de discrimination à l'égard des femmes, Haut-Commissariat des Nations unies aux droits de I'homme, http://www.ohchr.org/rrench/law/cedaw.htm. 
mains, ne sont pas appropriés 57 ». Depuis de nombreuses années, aucun des textes élaborés, " ni sur le plan européen, ni à l'ONU, ne fait plus aucune référence à cette convention 58 ». C'est notamment le cas de la convention de Palerme. En outre, les réglementaristes prétendent que le protocole additionnel contre la traite la remplace. Ce protocole qui condamne les trafiquants ne condamne aucunement les proxénètes. L'enjeu peut se résumer ainsi : «Faire disparaître cette convention [abolitionniste], c'est donc faire disparaître à la fois le jugement éthique sur le système prostitutionnel, le lien entre la traité et la prostitution et la condamnation pénale du proxénétisme ${ }^{59}$. "

À l'évidence, la convention abolitionniste est aujourd'hui un enjeu politique international important Les réglementaristes tentent de la délégitimer. Soulignons que, depuis la convention abolitionniste de 1949, la convention de Palerme est le premier texte juridique international qui se penche sur la question de la traite des êtres humains. Il importe donc de le juger par rapport à cette convention.

Selon la convention abolitionniste de 1949, la prostitution est une atteinte à la dignité humaine et doit être combattue en tant que telle: il faut cesser de criminaliser les personnes prostituées, qui sont des victimes du système prostitutionnel et non ses responsables et des criminelles, et réprimer ceux qui exploitent leur prostitution, les proxénètes : l'État ne doit ni imposer de règlements sur les personnes prostituées, ni surveiller celles-ci ; la prostitution est le socle sur lequel se développe la traite des êtres humains - combattre la traite sans en combattre sa cause, la prostitution, est un cul-de-sac - ; le consentement ou l'absence de consentement dans la prostitution est une question non pertinente car, comme pour l'esclavage ou le colonialisme, c'est l'existence même de l'institution qui pose problème: l'État est responsable de la prévention de la prostitution et de la trai-

57 Texte publié par la Fondation Scelles dans Peuple de Vabîme. La prostitution aujourd'hui, actes du colloque, UNESCO, Paris, 16 mai 2000, http://www.fondationsceËes.org/pages_html/ACTESDUCOLLOQUE.htm.

58 M.-V. Louis, «Prostitution. Contre l'Europe proxénète, la France peut-elle encore s'affirmer abolitionniste?», Les Cahiers du GREP Midi-Pyrénées, nos 25-26, 2001-2002, p. 135-178. Disponible sur le site www.marievictoirelouis.net/. 
te à des fins de prostitution ainsi que de la mise sur pied de programmes en faveur des personnes prostituées qui veulent quitter cette activité. Les États s'engagent en outre à protéger toutes les victimes de la traite.

Soixante-quatorze pays ont ratifié la convention de 1949. Certains, comme le Cambodge et la République tchèque sont des lieux importants de prostitution et de traite à des fins de prostitution. Les lois, même les meilleures, ne sont pas nécessairement appliquées. Cela est d'autant plus vrai que les traités internationaux rédigés avant 1960 ne sont pas dotés de [150] mécanismes de contrôle 60 . Contrairement aux traités subséquents, les pays adhérents qui ne respectent pas leurs engagements ne subissent ni remontrance ni sanction de la part des instances internationales 61 . Ne sont pas non plus appliqués les articles de la convention de 1949 qui demandent aux États adhérents de communiquer au secrétaire général de l'ONU « leurs textes de lois ou réglemente». En retour, ce dernier a l'obligation de publier «périodiquement» les renseignements reçus. Dans un rapport sur « la traite des femmes et des petites filles», le secrétaire général a déploré l'inexistence d'un organe de surveillance et d'un mécanisme d'application de la convention. Il observe «qu'il serait peut-être opportun d'envisager la possibilité de réviser ledit instrument afin de le rendre plus efficace en augmentant le nombre des États parties et en créant

60 C'est également le cas des conventions relatives à l'esclavage qui ont été adoptées par la Société des Nations en 1926 et par les Nations unies en 1949 et en 1956. Un rapport rédigé par David Weissbrodt et la Société anti-esclavagiste internationale, Abolir l'esclavage et ses formes contemporaines (New York et Genève, Haut-Commissariat des Nations unies aux droits de l'homme, 2002), souligne que les instruments interdisant l'esclavage et les pratiques analogues à l'esclavage ne prévoient aucune des procédures qui sont à présent considérées comme indispensables pour assurer le suivi du respect des obligations en matière de droits humains. Cela a pour conséquence que les instruments internationaux relatifs à l'esclavage ne désignent aucun organe conventionnel pour recevoir des rapports et les examiner. Ils ont peu d'effet quant au respect par les États des obligations qu'ils ont contractées et ne prévoient aucun mécanisme effectif de mise en oeuvre des dispositions qu'ils contiennent.

61 M. Marcovich, art. cité, p. 457 et suiv. 
un organe chargé d'examiner périodiquement des rapports 62 ». Dans un rapport pour la conférence mondiale des femmes de Pékin, le secrétaire général de l'ONU signale que la convention de 1949 «n'a eu que peu d'impact sur l'élimination de la traite des femmes à des fins sexuelles 63 ».

Du point de vue réglementariste, cette convention a le défaut de lier la traite et la prostitution, de condamner la prostitution, et de considérer les personnes prostituées, qu'elles soient recrutées au pays ou à l'étranger, comme des victimes du système prostitutionnel et non comme des criminelles. La convention abolitionniste limite toutefois sa portée à l'abolition des règlements qui pèsent sur les personnes prostituées et à la pénalisation des proxénètes au lieu de lutter pour l'abolition proprement dite de la prostitution et, en conséquence, elle oblitère la question de la responsabilité des prostitueurs dans la prostitution des personnes, acceptant de facto leur impunité. Néanmoins, ce texte de droit international pose, dans son préambule, un jugement politique et éthique fondamental sur la prostitution: «La [151] prostitution est incompatible avec la dignité et la valeur de la personne humaine. » Cette convention reste donc un obstacle majeur pour les réglementaristes qui la considèrent ajuste titre comme une entrave à la libéralisation du système proxénète.

\section{L'offensive réglementariste}

Retour à la table des matières

C'est à partir des années 1980, sous l'influence des Pays-Bas, que s'engage une mobilisation internationale visant à distinguer la traite de la prostitution, ainsi que la traite " forcée » de la migration volontaire des «travailleuses du sexe». En 1984, est créé à Amsterdam

62 Nations Unies, Traite des femmes et des petites filles. Rapport du Secrétaire général A/51/309,1996, § 56.

63 Projet de plate-forme d'action. Rapport du Secrétaire général, E.CN.6./1995/2, 27 février 1995. Ce texte a été amendé après la 39e commission du statut de la femme qui s'est tenue à New York en février. Il a donné lieu à un dernier texte intitulé : WCW Draft Plateform for Action, 24 mai, 1995, A/CONF.177./L.1. 
I'International Committee for Prostitutes Rights. En 1985, lors d'un congrès au Parlement européen, est présentée la charte mondiale des travailleuses du sexe qui revendique la décriminalisation de l'ensemble des activités du système prostitutionnel, y compris le proxénétisme. En 1987, on crée la fondation néerlandaise contre le trafic des femmes, Stichting tegen Vrouwenhandel (STV), laquelle entend s'opposer à la seule coercition subie par les « travailleuses du sexe » migrantes. Cette fondation est à l'origine de l'European Network against Trafficking in Women (1991) et du GAATW (1994). En 1991, a lieu un séminaire au Conseil de l'Europe, préparé et financé par les Pays-Bas, "contre la traite et la prostitution forcée». Les Pays-Bas, qui se cherchent des alliés, financent de nombreuses ONG sur les droits humains ou sur ceux des femmes. "Cette aide [est] conditionnelle à l'adoption des positions politiques néerlandaises $64 », c^{\prime}$ est-à-dire en faveur de la libéralisation de la prostitution.

La première dérive institutionnelle internationale en faveur de la libéralisation du système prostitutionnel mondial s'est manifestée en 1995 lors de la quatrième conférence mondiale de l'ONU pour la femme, tenue à Pékin, où l'on voit apparaître pour la première fois le principe de prostitution «forcée» dans une conférence internationale, ce qui sous-entend que seule la contrainte dans la prostitution doit être réprouvée. En 1997, sous la présidence néerlandaise, les lignes directrices issues de la conférence interministérielle de La Haye font apparaître une définition de la traite, où seul compte la preuve de la force, de la contrainte et de la menace. La même année, l'organisation AntiSlavery International publie un rapport prônant une redéfinition de la prostitution comme un «travail du sexe» sur l'agenda international 65. En 1998, I'OIT appelle dans un rapport à la reconnaissance économique de l'industrie du sexe. Sous le prétexte d'adopter une approche pragmatique, ce rapport considère qu'il est avantageux de reconnaître, de réglementer et de taxer l'industrie du sexe, afin de « cou-

64 M. Marcovich, art. cité, p. 470.

65 Anti-Slavery International, Redefining Prostitution as Sex Work on the International Agenda, Londres, Anti-Slavery International, 1997. 
vrir nombre d'activités lucratives qui y sont liées 66 ». En juin 1999, I'OIT adopte [152] la Convention contre les formes intolérables de travail pour les enfants 67 . Parmi la longue liste dressée se trouve la prostitution, reconnue pour la première fois dans un texte international comme un travail. Le rapport du rapporteur spécial sur les violences faites aux femmes à la Commission des droits de I'homme de I'ONU, en avril 2000, à Genève, affirme que la définition du trafic devait exclure les femmes "professionnelles du sexe migrantes illégales 68 ». En 2001, par la voix de son bureau en Asie du Sud-Est, l'Organisation mondiale de la santé (OMS) appelle à la légalisation de l'industrie du sexe afin de réduire l'épidémie de sida, réintroduisant ainsi l'argument hygiéniste qui a été le prétexte à la réglementation de la prostitution en Europe au dix-neuvième et au cours de la première moitié du vingtième siècle.

Selon certains tenants de la légitimation du «travail du sexe», l'instrument législatif de 1949 se « limitait uniquement» à la traite des femmes aux fins de prostitution et « délaissait la protection des enfants 69 ». Mais au même moment, la Convention relative aux droits de l'enfant qui demande aux États « d'empêcher » la vente, la prostitution et la pornographie mettant en scène des enfants 70 , est interprétée comme une acceptation de la prostitution «adulte». Tous conviennent que l'« exploitation sexuelle des enfants à des fins commerciales » est inacceptable. Or, les campagnes internationales contre cette « exploitation sexuelle commerciale » ne ciblent pas la prostitution et la pornographie comme les causes essentielles de cette « ex-

66 L. Lam Lim, Hie Sex Sector. The Economic and Social Bases of Prostitution in Southeast Asia, Geneva, ILO, 1998.

67 www.ilo.org/public/english/employment/skills/hrdr/iiistr/c_182.htm.

68 Economic and Social Council, United Nations, Integration of the Human Rights of Women and the Gender Perspective. Violence against Women, Report of the Special Rapporteur on violence against women, its causes and consequences, Ms. Radhika Coomaraswamy, on trafficking in women, women's migration and violence against women, submitted in accordance with Commission on Human Rights resolution 1997/44, E/CN.4/2000/68, 29 février 2000.

69 P. Boonpala et J. Kane, Le trafic des enfants dans le monde. Problèmes et réponses, Genève, Bureau international du travail, 2001.

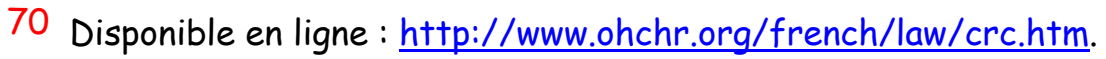


ploitation », mais restreignent le problème à l'âge de consentement. Avant cet âge, il est interdit d'utiliser des enfants dans la pornographie et la prostitution, après cet âge, pour les réglementaristes, les jeunes adultes sont « libres» de «se prostituer» ou de tourner dans une production pornographique.

Enfin, l'article 6 de la CEDEF, qui a pourtant pour origine directe la convention abolitionniste, est interprété par les membres du Comité contre la discrimination à l'égard des femmes, l'organe de I'ONU chargé de suivre l'application de la CEDEF, comme relevant de la «prostitution forcée 71 ». La condamnation dans la convention de 1949 de «l'exploitation de la prostitution» [153] d'autrui, c'est-à-dire du proxénétisme, a été transformée au cours de la dernière décennie en condamnation de l'«exploitation» dans la prostitution, laquelle est nécessairement liée à l'usage de la force ou de la contrainte.

Les promoteurs de la prostitution d'autrui ont réussi à imposer un nouveau vocabulaire qui structure grandement l'opinion publique internationale et les politiques publiques. Outre l'introduction du concept de "prostitution forcée», les mots ont été détournés de leur sens, voire inversés. Les industries du sexe sont des « industries du loisir » (pour qui ?): la prostitution est rebaptisée "travail du sexe» ou «vente de services sexuels» (ou encore «érotiques» et «sensuels»), elle est devenue une " sexualité récréative » (pour qui ?); les proxénètes sont des « managers», des « entrepreneurs érotiques » ou de simples "gens d'affaires»; les bars de danse nue et les maisons closes sont des « lieux de divertissement», des «établissements érotiques» ou des « entreprises de relaxation 》 (pour qui ?). Les «travailleuses du sexe» «érotiques» ou «exotiques» obtiennent des «visas d'artiste». La personne prostituée est même, à l'occasion, considérée non plus comme une «travailleuse du sexe», mais comme un « entrepreneur», son corps étant un « capital » à faire fructifier. Ils ont introduit le concept d' $d$ exploitation sexuelle » pour remplacer

71 Heisoo Shin (experte coréenne auprès de I'ONU pour l'application de la CEDEF), «EI CEDAW y los Derechos Humanos de las mujeres : protección des las mujeres y niñas contra la explotación sexual », Congreso international Derechos humanos y prostitución, Madrid, 22 y 23 de noviembre de 2006, Madrid, Área de Gobierno de Empleo y Servicos a la Ciudania, 2007, p. 158-167. 
le terme «prostitution», étant entendu qu'il n'y a plus d'« exploitation sexuelle »lorsqu'il y a « consentement ».

D'un côté, le vocabulaire est essentiellement économique : on y parle de travail, d'exploitation, de demande, de vente et d'achat de services sexuels, de clients ou de consommateurs, de managers ou de chefs d'entreprise, de trafic, etc. En faisant ainsi de la prostitution un phénomène stricto sensu économique, on fait l'impasse sur la prostitution comme forme et moyen de la domination masculine, laquelle réduit les femmes à un sexe, qui se voit, en outre, transformé en marchandise. D'un autre côté, on fait appel aux «droits humains», en créant le «droit de se prostituer», lequel ne relèverait que d'un choix strictement individuel.

Les gouvernements favorables à la réglementation de la prostitution et à la légalisation du proxénétisme soutiennent dans un même mouvement la criminalisation des «travailleuses du sexe » migrantes illégales et de celles qui refusent l'enfermement en bordels ou dans les zones de «tolérance». Ils refusent la notion de «victime» dans le cas du trafic et amoindrissent sérieusement sa portée dans le cas de la traite. Par conséquent, les personnes arrivées dans le pays par la traite ou le trafic sont considérées comme des criminelles plutôt que comme des victimes d'un crime; elles sont une menace contre l'intégrité territoriale de l'État ainsi que contre sa sécurité.

$\grave{A}$ première vue, la reconnaissance de la prostitution comme travail semble une avancée par rapport à la situation antérieure, puisque les personnes prostituées obtiendraient des droits qu'elles n'auraient pas autrement. Toutefois, pour obtenir ces droits, les personnes prostituées d'Allemagne, dont le nombre est estimé officiellement, en mars 2006, à quatre cent mille, doivent signer un contrat de « travail » avec les propriétaires d'un bordel ou d'un eros center. Selon le gouvernement allemand, seulement $1 \%$ d'entre elles [154] ont signé un tel contrat. Or, historiquement, on constate que les maisons closes agréées n'empêchent pas la prostitution de rue et les clandestines sont nettement plus nombreuses que les inscrites. En France, avant 1946, année de la fermeture des mille cinq cents maisons closes officielles, on estimait qu'une femme prostituée sur cinq était en bordel, 
et une sur quatorze seulement n'était pas une « insoumise », c'est-àdire était enregistrée 72 .

\section{En guise de conclusion}

Retour à la table des matières

Les études récentes sur le système légal de la prostitution en Australie et au Nevada démontrent que la prostitution réglementée se fait au détriment des personnes prostituées 73 .

La réglementation de la prostitution et la légalisation du proxénétisme donne une impulsion importante à l'industrie prostitutionnelle et, par conséquent, à la traite à des fins de prostitution. Aux Pays-Bas, $80 \%$ des personnes prostituées sont originaires de l'étranger et $70 \%$ d'entre elles sont sans papiers. Les proportions sont similaires, malgré quelques variations mineures, en Allemagne, dans la majorité des Lander d'Autriche, en Suisse, en Grèce, en Australie et en NouvelleZélande.

Il y a historiquement une forte corrélation entre les politiques libérales de laisser-faire et leur équivalent moderne, le néolibéralisme, la croissance de l'industrie de la prostitution ainsi que de son corollaire, la traite des femmes et des enfants à des fins de prostitution, et le réglementarisme (première et deuxième vague). Moins l'État intervient pour contrer les effets producteurs d'inégalités et de pauvreté du capitalisme, plus il intervient pour enfermer les femmes, victimes d'une marchandisation sexuelle, dans des bordels réglementés, dont il tire d'importants bénéfices sous forme de taxes et d'impôts. $A$ contrario, plus les États interviennent pour limiter la production d'inégalités et de pauvreté, moins les femmes et les enfants sont victimes de l'industrie du sexe, moins leur corps et leur sexe sont soumis au

72 A. Corbin, Les filles de noce. Misère sexuelle et prostitution au XIXe siècle, Paris, Flammarion, 1982.

73 M. L. Sullivan, Making Sex Work: A Failed Experiment with Legalised Prostitution, North Melbourne, Spinifex, 2007 ; M. Farley, Prostitution and Trafficking in Nevada: Making the Connections, San Francisco, PRE, 2007. 
marché du plaisir masculin. Aujourd'hui, dans un monde où règnent les valeurs libérales, comme à l'époque de la première industrialisation et des politiques de laisser-faire économique, la prostitution des femmes et enfants est un phénomène de masse.

Les arguments ont changé: I'hygiénisme de la première réglementation a laissé place au « droit» des personnes de « se prostituer »... au plus grand profit des proxénètes et des prostitueurs.

Fin du texte 